\title{
Challenges to Medical Education on Surgical Services During the COVID-19 Pandemic: a Medical Student's Perspective
}

\author{
Benedict Reed $^{1}$
}

Accepted: 6 November 2020 / Published online: 7 January 2021

(C) International Association of Medical Science Educators 2021

\section{Dear Editor,}

It is with a great deal of optimism that I read the article by Schaffir et al., outlining the challenges that COVID-19 poses to the provision of an adequate surgical education for medical students [1].

As a final year medical student at Imperial College London, and a hopeful future surgeon, the pandemic has certainly had an adverse effect on my education. As life tends, seemingly asymptotically, back to normality, we'd be remiss to not take this opportunity to modernize surgical education. We shouldn't be looking for solutions to meet our old standards but for those that exceed them.

The exposure to real-life surgical cases in theaters is invaluable. In looking forward, it might be helpful to look to surgery's past. Why shouldn't we embrace a modern analogue to the surgical galleries that were previously integral? Surgical lights already have cameras for capturing footage for posterity. From this perspective, the scrupulous nature of surgery, often downplayed in student's didactic education, could be visualized in real time. Why couldn't students consent patients and then witness surgeries completely virtually? My medical school plans to use the Microsoft HoloLens, worn by a professor in clinic, to allow students to witness clinical examinations virtually, in preparation for our finals this winter. This format sets a precedent that might soon allow students to "drop-in" to the eyes of a surgeon.

Streaming surgeries is an ethically complex but technologically simple task. More advanced systems, like the VR experiences mentioned in this paper, remain prohibitively expensive. However, with surgical rotations making up on

Benedict Reed

benedict.reed@nhs.net

1 Imperial College School of Medicine Charing Cross Hospital, London, UK average just $29 \%$ of clinical training and the ever-falling price of VR headsets, institutions could employ the use of a small number of these devices [2]. VR sessions would compensate for the deleterious effect of COVID on students' exposure to surgery.

The effects of COVID-19 have been overwhelmingly negative. One immensely positive consequence is that as our systems for remote learning improve, the implicit barriers to inter-institution collaboration fade away. For example, students from my university are now able to "attend" nighttime Orthopedics lectures at King's College. This culture of collaboration simply did not exist for our predecessors and its impact should not be understated.

While the pandemic has certainly created a great deal of disruption to the education of medical students, COVID-19 has accelerated the introduction of new teaching methods and I'm hugely hopeful for the future of surgical education.

\section{Compliance with Ethical Standards}

Conflict of Interest The author declares that there is no conflict of interest.

Ethical Approval NA

Informed Consent NA

\section{References}

1. Schaffir J, Strafford K, Worly B, et al. Challenges to medical education on surgical services during the COVID-19 pandemic. Med Sci Educ. https://doi.org/10.1007/s40670-020-01072-2. 2020.

2. Vaidya HJ, Emery AW, Alexander EC, et al. Clinical specialty training in UK undergraduate medical schools: a retrospective observational study. https://doi.org/10.1136/bmjopen-2018025403.

Publisher's Note Springer Nature remains neutral with regard to jurisdictional claims in published maps and institutional affiliations. 